\title{
Informed Consent under the Ghana Health Service Patients Charter: Practice and Awareness
}

\author{
Alexander Acheampong Oti ${ }^{1}$, Ernest Owusu-Dapaah ${ }^{2}$, \\ Chris Adomako-Kwaakye², Daniel Kwesi Sabbah', \\ Solomon Obiri-Yeboah', Ama Amuasi' ${ }^{1}$, Adu Tutu Amankwa3 , \\ Ebenezer Adjei-Bediako², Eva Adu-Boakye4 \\ ${ }^{1}$ Dental School, Kwame Nkrumah University of Science and Technology, Kumasi, Ghana \\ ${ }^{2}$ Faculty of Law, Kwame Nkrumah University of Science and Technology, Kumasi, Ghana \\ ${ }^{3}$ School of Medical Sciences, Kwame Nkrumah University of Science and Technology, Kumasi, Ghana \\ ${ }^{4}$ Family Medicine Directorate, Komfo Anokye Teaching Hospital, Kumasi, Ghana \\ Email: aotiacheampong@yahoo.com
}

Received 24 February 2016; accepted 19 April 2016; published 22 April 2016

Copyright (C) 2016 by authors and Scientific Research Publishing Inc.

This work is licensed under the Creative Commons Attribution International License (CC BY). http://creativecommons.org/licenses/by/4.0/

(c) $\underset{\text { EY }}{ }$ Open Access

\section{Abstract}

Background: Every human being of adult years and sound mind has a right to determine what shall be done with his/her own body. Patient autonomy and the practice of informed patient consent are now pivotal in medical practice. Aim: To assess patient's knowledge of Patients' Rights Charter and whether patients receive adequate information to enable them make an informed consent to a particular treatment. Methodology: Patients who were undergoing elective surgery from selected surgical departments of Komfo Anokye teaching hospital in Kumasi were randomly selected and assisted to answer structured questionnaire without the knowledge of their doctors. The study period was in June to December (2014). Descriptive analysis was done using SPSS (II) of the results. Results: $84.7 \%$ (144) had no idea about the Patients' Rights Charter of the Ghana Health Service. $75 \%$ (128) did not know or had not heard of informed patient consent. Of those who knew of the charter, $85 \%$ (37) had ever stayed in a developed country. $60 \%(102)$ did not know of their diagnosis. $79 \%$ (134) said the doctor only asked them to either sign or thumb print the consent document without giving them treatment options or possible complications. Conclusion: Most of respondents undergoing various surgical procedures at the Komfo Anokye Teaching Hospital were not aware of the existence of the Patients' Rights Charter of the Ghana Health Service. Again, practitioners did not provide sufficient information to patients for them to make an informed decision about their health. 
Keywords

Informed, Consent, Patient, Charter, Autonomy

\section{Introduction}

The acquisition of patient's informed consent before undergoing any treatment is a complex process. It is increasingly recognized that the patient plays an active role rather than just passively accepts doctors' advice. Informed consent is a legal doctrine in medical practice. It has been argued prima facie that every competent adult has the right to decide whether to consent or refuse any medical treatment, even if such refusal could lead to the death of that patient [1]. Recipients of health care have specific rights with regard to its delivery. Within this context, the providers of health care, should also be knowledgeable of and have respect for those rights.

At the basis of the patients' rights are autonomy and informed consent. An optimally informed patient will have more realistic expectations regarding a surgical procedure and its associated risks. Well-informed patients will be more satisfied and file fewer legal claims [2].

Informed consent is a voluntary un-coerced decision made by a competent autonomous individual to accept or reject some proposed courses of action. Patients have the right to be informed about their diagnosis and treatment. The patients having received the relevant information about their condition which can now give consent to the health care provider before any treatment regime which can begin [3].

The doctrine of informed consent within the context of physician-patient relationships goes far back into English common law. As early as 1767, doctors were charged with the tort of "battery" (i.e., an unauthorized physical contact with a patient) if they had not gained the consent of their patients prior to performing a surgery or procedure (e.g., Slater v. Baker and Stapleton) [4].

A patients right to autonomy and informed consent during medical treatment was popularized as a legal doctrine by Cardozo J. in the Schloendorf case [5] where he said that "every human being of adult years and sound mind has the right to determine what shall be done with his own body, and a surgeon who performs surgery without patients content commits and assault for which he is liable in damages”.

The consent form should not be confused with the consent process; the form just documents that the process has occurred. But the process must meet the local or international standard to make it valid. In other words, the signed form is not valid if the process involved is inadequate.

Requiring that the patient first consented was only half the task. The other half involved the patient's receipt of sufficient information upon which to make a sound decision. Thus, the concept of informed consent is developed on the premise of two distinct components: a person's inherent right to determine what happens to his or her body and a doctor's inherent duty to provide a person with enough information so as to ensure that the patient's ultimate decision is based on an appreciable knowledge of his/her condition, the available options for treatment, known risks and prognoses. This is known as the patient autonomy [6]. This means that the patient does not have a duty to inquire about risks or options; the duty rests with the treating doctor. The concept of informed consent is perhaps more closely related to surgical specialties than other clinical disciplines because of the necessity for patients' decisions to participate in surgery and their authorization of surgeons to operate on them [7].

In Ghana, our medical laws are not well developed and in the areas where they exist they have not being adequately tested in court of competent jurisdiction. The patient's right charter has being adopted by the Ghana Health Service and is now a law for all health care practitioners to abide by it. A key component of this document is the concept of informed patient consent.

\section{Aim}

To assess patients' knowledge of Patients Right Charter and to evaluate the quality of informed consent obtained by doctors to ascertain whether patients received adequate information to enable them make an informed choice to a particular treatment.

\section{Method/Statistics}

The study period was from June to December (2014). Consecutive patients who were undergoing elective sur- 
gery from selected surgical departments of Komfo Anokye Teaching hospital in Kumasi were randomly selected and assisted to answer structured questionnaire without the knowledge of their doctors. Patients were interviewed at the main theatre preoperative area of KATH. Those who consented to be part of the study were 170 . The inclusion criteria included adult age of medical consent in Ghana (18 years and above), competent and of sound mind. The factors which could affect the knowledge of Patients' Rights Charter were not investigated in this study.

All emergency surgeries were excluded. Data was entered in SPSS (II) for descriptive analysis.

\section{Results}

The total number of patients was 170 during the study period. The male to female ratio was 2:1.

84.7\% (144) have no idea about Patients Right Charter from Ghana Health Service.

$75 \%$ (128) do not know or have not heard of informed patient consent.

Those who knew of the charter, 85\% (37) have ever stayed in developed country. 60\% (102) do not know of their diagnosis.79\% (134) said the doctor only to them they are due for surgery asked them to either sign or thumb print the consent document without giving them treatment options or possible complications. 58\% (98) want to be part of the decision process in their treatment. $42 \%$ (76) think that doctors know the best, so should choose the treatment for them.

\section{Discussion}

A patient who is given treatment without informed consent may claim compensation at law if the risk materializes. It is no defense that all other aspects of care were given with reasonable care. Every adult human being of sound mind has a right to determine what shall be done with his own body and he/she has the right and responsibility to make health care decision [5].

In this current study, majority of the respondents were educated with the majority have attended either junior high or middle school education, but as to whether they could read and write English which is the national language was not evaluated since most of the respondents (65\%) asked the interviewer to read and explain the content of the questionnaire in the local language (Twi). Majority of respondents had no idea of the patient's right charter from the Ghana Health Services and the concept of informed patient consent (84.7 and 75.0 respectively). This finding is similar to a study of a black population in South Africa [8]. In that study it was stated that knowledge and practice of informed consent is light years away from most of the black population in South Africa.

In this study, it was evident that $79 \%$ of respondents said that doctors either asked them to sign or thumbprint the consent form without any explanation as to the content of the form. It was therefore not surprising that majority of patients undergoing surgical treatment did not even know of their diagnosis and were only made to either sign or thumbprint a document most likely to be the consent form

In a related study from Nigeria, it was shown that most surgeons fall short in providing necessary information to patients about their illnesses when obtaining consents for surgical interventions [9].

The Nigerian study acknowledged poor communication as mostly responsible for patients' refusal of necessary surgical procedures [10].

This practice does not constitute adequate disclosure of information to the patient. From the above results it was implied that patients did not receive adequate information for them to make any informed choice about their treatment. This in our view constitutes consent by insufficient consent process. The patient actually signed the consent form without going through the consent process adequately.

The practice of insufficient disclosure in this present study could be due to that fact that most practitioners do not know much about the process of the informed consent and the consequence of insufficient disclosure. In a South African study [11], doctors were asked to rank a series of potential challenges experienced while obtaining informed consent in practice; the reasons given were languages differences (87.5\%), time constraints (86.9\%), high work load (85.0\%), and lack of education on the part of patients (84.5\%). The challenges experienced in the South African study might not be different from Ghana which has multiethnic society, very low doctor to patient ratio and the fact that most of the respondents in this study could not read and answer the questionnaire by themselves. It is difficult to explain medical diagnosis, treatment and complications in our various local languages to patients and this may be a contributing factor to inadequate disclosure to patients.

An article in the Ghana Medical journal [12] concluded that there must be full disclosure of relevant informa- 
tion to the patients to enable them make an informed consent and practitioners cannot rely on the concept of therapeutic privilege. The argument of the above article was supported by the following decided cases: In Meyers Estate et al. v Rogers (1991) [13], a 37-year old woman died after intravenous injection of a contrast medium for a routine radiologic procedure. The physician intentionally withheld information about the risks associated with contrast media. The Ontario court rejected the radiologist's claim of therapeutic privilege as a defence against failing to warn the patient of the risks of intravenous contrast medium injection. In the above case, the court cited the case of Reibl v Hughes [14], to support its argument and stated that "the therapeutic privilege exception to the doctor's duty of disclosure should not be part of Canadian law because of its potential to erode informed consent”.

In order to prevail on a charge that a doctor performed a treatment or procedure without valid informed consent, the patient must usually show that, had he/she known of the particular risk, outcome, or alternative treatment allegedly not disclosed, the patient would not have opted for the chosen treatment or procedure and thus, would have avoided the risk. In other words, the patient must show a harmful consequence to the alleged failure to disclose.

Again $58 \%$ of patients from this study wanted to be part of the decision making concerning their care. Patients cannot be part of this important process if there is insufficient disclosure. The other reason that could explain the insufficient disclosure in our study could be due to high patient load and the fact that in Ghana some patient think the doctors know the best and they should go ahead and do what they think is in their best interest. This was also evident from this study where $52 \%$ of patients were of the view that doctors should go ahead and make the decision on their behalf. This phenomenon from patients is not so peculiar to Ghana. It is well known that some patients make decisions in a linear, rational fashion, considering specific risks and benefits, others make their decisions based on intuitions or instinct ("the tumor must come out, doc. I don't care what you tell me") [15].

These relatively justifiable excuses of lack of disclosure are still not valid for practitioners to rely upon to deny patient vital information about their condition.

In our view allowing these excuses will defeat the concept of patient autonomy in medical care. Doctors should in our view try and explain the consent procedure in very simple terms for their patients. Practitioners cannot assume the position of the patient as far as informed consent in medical practice is concerned.

Previous studies in postoperative patients have demonstrated the necessity for surgeons to spend more time and provide more information to their patient's preoperatively [16]. This is a surgeon's moral obligation and one way to avoid situations where patients are afraid or nervous before surgical operations.

\section{Conclusion}

This study has demonstrated that most of respondents undergoing various surgical procedures at the Komfo Anokye Teaching Hospital are not aware of the existence of the Patients Right Charter of Ghana Health Service. Again, practitioners do not provide sufficient information to patients for them to make an informed decision about their health which is a deviation from the Ghana Health Services Patients' Right Charter. Majority of patients want to be a part of the decisions concerning their health.

\section{Recommendation}

The basic problem is that most patients do not know about their right probably because they are not well educated about their health. Practitioners should have training in the consent process and how best to explain medical conditions in local languages. What then is the legal implication if the patient asks the doctor to do what they think is in their best interest? There must be clear guidelines from Ghana Medical and Dental Council to save health care providers from expensive litigations.

\section{Limitation of Study}

The factors which could affect the knowledge of Patients' Rights Charter were not investigated in this study. Further study will be needed to address the above. There could be a language barrier during the interpretation of the questionnaire into local language for those patients who could not read and write.

\section{References}

[1] Airedale NHS Trust v. Bland 1993, 2 WLR 316. 
[2] Leclercq, W.K.G., Keulers, B.J., Scheltinga, M.R.M., Spauwen, P.H.M. and van der Wilt, G.-J. (2010) A Review of Surgical Informed Consent: Past, Present, and Future. A Quest to Help Patients Make Better Decisions. World Journal of Surgery, 34, 1406-1415. http://dx.doi.org/10.1007/s00268-010-0542-0

[3] Goldberg, B.A. (1978) The Duty of Hospitals and Hospital Medical Staffs to Regulate the Quality of Patient Care. Western Journal of Medicine, 129, 443-451.

[4] Slater vs. Baker and Stapleton. Eng Rep (1767).

[5] Schloendorf v. Society of New York Hospit. 1914105 NE 92.

[6] Entwistle, V.A., Carter, S.M., Cribb, A. and McCaffery, K. (2010) Supporting Patient Autonomy: The Importance of Clinician-Patient Relationships. Journal of General Internal Medicine, 25, 741-745. http://dx.doi.org/10.1007/s11606-010-1292-2

[7] Angelos, P., Lafreniere, R. and Murphy, T.F. (2003) Ethical Issues in Surgical Treatment and Research. Current Problems in Surgery, 40, 353-356.

[8] Mhlongo, S.W. and Mdingi, G.V. (1997) Informed Consent Is Light Years away for Black African Patients. British Medical Journal, 315, 252.

[9] Ogundiran, T.O. and Adebamowo, C.A. (2012) Surgeon-Patient Information Disclosure Practices in Southwestern Nigeria. Medical Principles and Practice, 21, 238-243. http://dx.doi.org/10.1159/000333817

[10] Ogundiran, T.O. and Adebamowo, C.A. (2010) Surgeons' Opinions and Practice of Informed Consent in Nigeria. Journal of Medical Ethics, 36, 741-745. http://dx.doi.org/10.1136/jme.2010.037440

[11] Sylvester, C.C. (2013) Evaluating the Quality of Informed Consent and Comtemporay Clinical Practices by Medical Doctors in South Africa: An Empirical Study. BMC Medical Ethics, 14, 1-17.

[12] Edwin, A. (2008) Don’t Lie but Don't Tell the Whole Truth: The Therapeutic Privilege-Is It Ever Justified? Ghana Medical Journal 42, 156-161.

[13] Meyers Estate et al. v Rogers. 78 D.L.R. (4th).

[14] Reibl v, Hughes. 1980.

[15] Schneider, C.E. (1998) The Practice of Autonomy: Doctors, and Medical Decisions. Oxford University Press, New York, 35-75, 92-99.

[16] Osime, O.C., Okojie, O., Osadolor, F. and Mohammed, S. (2004) Current Practices and Medico-Legal Aspects of PreOperative Consent. East African Medical Journal, 81, 331-335. http://dx.doi.org/10.4314/eamj.v81i7.9186 